\title{
Development of a Novel Power-Monitoring System Using Internet and Wireless Sensor Network Technologies
}

\author{
Shih-Sung Lin, ${ }^{*}$ Chien-Wu Lan, Ping-Nan Chen, ${ }^{1}$ Min-Hsiung Hung, ${ }^{2}$ \\ Kuang-Hsiung Tan, Ya-Hui Wu, and Jo-Yen Nieh \\ Department of Electrical and Electronic Engineering, Chung Cheng Institute of Technology, \\ National Defense University, No. 75, Shiyuan Rd., Daxi Dist, Taoyuan, 335, Taiwan, R.O.C. \\ ${ }^{1}$ Department of Biomedical Engineering, National Defense Medical Center, \\ No. 161, Sec. 6, Minquan E. Rd., Neihu Dist., Taipei, 114, Taiwan, R.O.C. \\ ${ }^{2}$ Department of Computer Science and Information Engineering, Chinese Culture University, \\ Taipei 111, Taiwan, R.O.C.
}

(Received February 26, 2016; accepted May 15, 2017)

Keywords: wireless sensor network, power-monitoring devices, monitoring and control system

Based on Internet and ZigBee wireless sensor networks, in this paper, we describe a novel power-monitoring system architecture that makes the mobile deployment of power-monitoring points quite easy and applicable to diverse power-monitoring targets. Specifically, we have designed a novel power-monitoring server (PMS) to serve as the operational core of the proposed architecture. The PMS can monitor, record, display, and manage the power consumption information of general equipment and power facilities. It can also actively detect if the power consumption is over a preset threshold and promptly send warning messages to power management personnel through mobile phone messages and e-mails. In addition, we have developed six types of wire or wireless portable power-monitoring devices (PMDs) that can be used to monitor a variety of targets, such as general equipment, traditional distribution boxes, and new distribution boxes with digital meters and can be deployed in different environments. Finally, a paradigm power-monitoring system has been constructed to monitor the power consumption of the campus at Chung Cheng Institute of Technology, National Defense University, to validate the feasibility and practicality of the proposed PMS architecture. The research results in this paper provide an effective solution to the problem that the existing PMSs may not be applicable to different environments and did not take diverse monitoring targets into consideration.

\section{Introduction}

In recent years, owing to the industry maturing and accompanied by increased requirements of power consumption, improving the efficiency of power and finding new reusable energy still present many problems to be overcome. To reduce power consumption and help users know how power is used, various power-monitoring and control systems (PMCS) have been developed. ${ }^{(1-4)}$ The advantages of PMCSs can help companies reduce the cost of power and also allow them to make decisions about power efficiency on demand.

*Corresponding author: e-mail: shihsunglin@gmail.com

http://dx.doi.org/10.18494/SAM.2017.1598 
Nowadays, applications using the Internet have spread all over the world. Many Web-based PMCSs have been developed to assess the conditions of remote facilities via the Internet. ${ }^{(5,6)}$ Applications of ZigBee wireless sensor networks (ZWSNs) have grown in recent years owing to their benefits of low cost, low complexity, high scalability, high mobility, and low-power consumption. ${ }^{(7)}$ Their applications include power measurement, ${ }^{(8)}$ environmental data sensing, ${ }^{(9,10)}$ home appliance control, ${ }^{(11-13)}$ and automation control. ${ }^{(14,15)}$

Several literature articles related to PMCSs based on the Internet or wireless sensor networks are summarized in the following. Lin utilized LabVIEW and a single chip to gather harmonic waves from a power system to enable users to manage the system remotely using a graphic user interface. $^{(5)}$ Zhang and Li developed a remote power quality monitoring system to enable users to monitor and analyze power quality via the Internet. ${ }^{(6)}$ Luan et al. designed a data collection module integrated with ZigBee devices and a digital meter to gather power parameters and detect power failures for smart grid applications. ${ }^{(16)}$ Chen and Jiang developed two topologies for a ZigBee wireless sensor network of a power acquisition system that allowed the system to collect power data more efficiently at different distances. ${ }^{(17)}$ Hong and Park proposed a flexible powermonitoring device (PMD) with clamp-type sensors to allow users to obtain the working current of equipment without disassembling it. ${ }^{(18)}$ Itoh et al. developed a wireless current clamp probe integrated with a thermometer and a network system that enabled the simultaneous monitoring of power lines. ${ }^{(19)}$

By reviewing the literature related to PMCSs, we find that the existing PMCSs have the following drawbacks that need to be improved further.

(1) They do not enable the monitoring devices to be deployed in different environments.

Some monitoring areas already have Internet infrastructure; users can deploy PMDs with Ethernet interfaces easily. However, some places lack Internet infrastructure and have difficulty constructing the necessary infrastructure owing to limited budgets. Hence, the PMD should possess different communication interfaces to fulfill the requirement for deployment in different environments and to send power data to a power-monitoring server (PMS) in a simple manner.

(2) The PMD lacks the capability of monitoring diverse targets.

In a power-monitoring application, there may be various targets ready to be monitored, such as home appliances, industrial equipment, power distribution boxes, and equipment with digital meters. Most current applications use one type of PMD to satisfy their specific needs and are deficient in designing diverse PMDs to handle various kinds of monitoring targets.

(3) Few literature articles mention how to enhance the reliability and improve the transmission efficiency of ZWSN.

If packet losses occur in the process of wireless transmission, management personnel may misjudge the power consumption of the monitoring targets. Thus, ensuring the reliability of wireless data transmission is important to the construction of ZWSN-based MC systems. In addition, the maximum throughput of a ZWSN is only $250 \mathrm{Kbps}$. When the number of deployed ZigBee nodes is large, a coordinator may require a long period of time to collect data from all of the sensor nodes, thereby limiting the practicality of the PMCS. Therefore, an efficient data collection scheme is fundamental to a ZWSN-based MC system.

In this paper, we address some of the problems existing in current PMCSs. We summarize the topics in this paper as follows: 
- Design a novel architecture for PMCSs to solve the shortcomings of existing systems.

- Design key functional components into the PMS as the operational core of the PMCSs to meet the requirements we identified.

- Develop diverse portable PMDs suitable for deploying in different environments for various monitoring targets in an easy way.

\section{Architecture of the Power-Monitoring System under Study}

Figure 1 shows the architecture of the power-monitoring system under study. The structure consists of three parts: the equipment side, the server side, and the client side, are illustrated in the figure.

\section{- Equipment side}

The design concept of the equipment side is to allow the PMS to be applicable to various monitored equipment. We distinguish the equipment into three types and illustrate as follows:

(1) General equipment

General equipment includes air conditioners, microwave ovens, and home appliances with high power consumption, which have a socket connecting to a power supply.

(2) Power distribution box (PDB)

The PDB is usually installed in a room to provide power to fulfill users' needs. The PDB typically has several no-fuse breakers in it to distribute power to sockets inside a room.

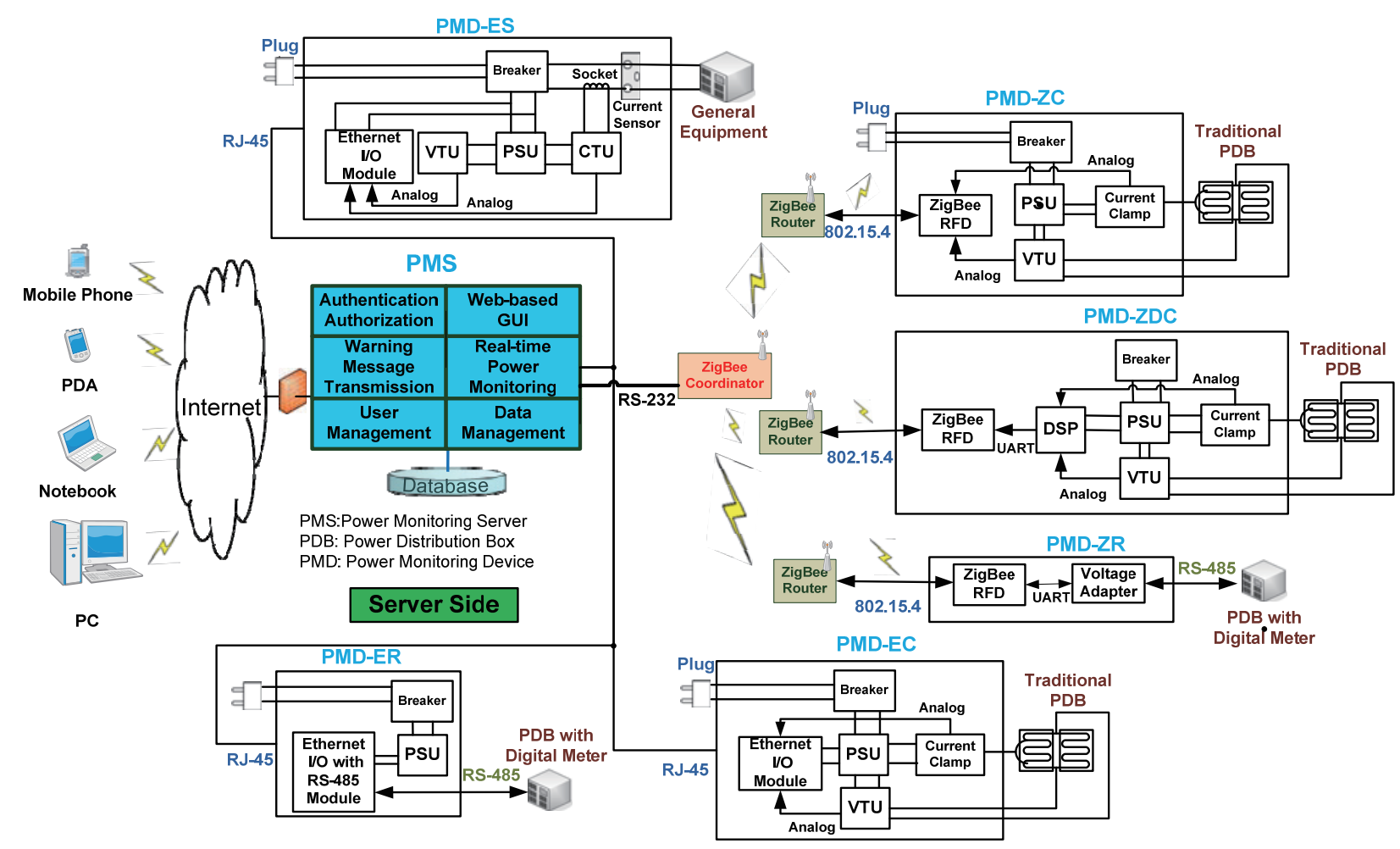

Fig. 1. (Color online) Architecture of the power monitoring system. 
(3) PDB with digital meter

This kind of PDB is embedded with a digital meter that can gather power parameters online and usually has an RS-485 interface for data exchanging with personal computers. Thus, users can obtain precise power information and understand the patterns of use of power-consuming appliances.

In this study, we designed six types of PMDs that can be deployed in diverse environments and to monitor various targets. A brief introduction to PMDs follows and the details of their design are given later.

(1) PMD-ES (PMD with Ethernet and socket)

The PMD-ES has a socket that allows the monitored equipment to be plugged in for supporting power and acquiring power consumption parameters such as current and voltage. An Ethernetready I/O module was designed inside the PMD-ES to send data to the PMS. Users can measure the power consumption without disassembling the monitored equipment and changing its circuits. Hence, PMD-ES is suitable for use with general equipment.

(2) PMD-EC (PMD with Ethernet I/O and current clamp)

A PMD-EC equipped with a current clamp can clasp on a power line in a PDB to detect current while the equipment is working. Then, the PMD-EC can send the power parameters to the PMS through the Internet (or Intranet) via a wired connection.

(3) PMD-ER (PMD with Ethernet I/O and RS-485)

A PMD-ER has an RS-485 interface to the power parameters from a digital meter embedded in a power station and an Ethernet interface to provide the ability to exchange internet data with the PMS.

(4) PMD-ZC (PMD with ZigBee and current clamp)

The structure of a PMD-ZC is similar to that of the PMD-EC but different in the communication mode. The PMD-ZC adopts a ZigBee wireless sensor network as the communication interface to send the power data to the PMS wirelessly.

(5) PMD-ZDC (PMD with ZigBee, DSP, and current clamp)

A PMD-ZDC has a current clamp, a digital signal process (DSP) module, and a ZigBee device to acquire the working current of equipment, compute power quality, detect abnormal conditions, and transmit power parameters over the air to the PMS. Similar to the PMD-EC, a PMD-ZDC is suitable for deploying in the PDB.

(6) PMD-ZR (PMD with ZigBee and RS-485 adapter)

A PMD-ZR consists of an RS-485 adapter and a ZigBee device. It uses an RS-485 interface to collect power parameters from the digital meters inside a PDB and send the data back to the PMS wirelessly.

- Server side

On the server side, we designed a PMS as the operational core of the whole PMS framework. Several functional components were designed in the PMS to enable the user to operate the necessary functions for power management, including real-time power monitoring, data management, warning message transmission, user management, authentication and authorization, and Web-based graphical user interface (GUI). The PMS possesses an Ethernet interface as well as an RS-232 interface connecting with the ZigBee coordinator to provide both wired and wireless communication capabilities. The power parameters gathered from the PMD are saved into the system's database. When the PMS detects an abnormal condition of the working equipment, it sends an alarm message to the responsible user to deal with the situation. 


\section{- Client side}

On the client side, users can use the browser in devices such as a PC or a notebook to operate the system functions via the Internet.

\section{Design of PMDs}

\subsection{Design of PMD-ES}

The design concept of the PMD-ES is to allow a user to collect the power consumption data from monitored equipment without changing or modifying the circuit inside it. The functional blocks are shown in Fig. 2. A PMD-ER consists of a socket, a plug, a current sensor, a breaker, a current transformation unit (CTU), and a power supply unit (PSU). After the current sensor measures an AC current, the CTU transforms the measured AC current into a DC voltage. The Ethernet-ready I/O module can then acquire the output DC voltage of the CTU through its analog input channel. The breaker is the power switch of the PMD-ES. It protects against short circuits. The PSU transforms the AC power to provide the DC power needed by the CTU. With such a design, the equipment's working current can be conveniently measured by just putting the equipment's plug in the socket of the PSDM and inserting the plug of the PSDM into the wall socket. This makes the installation of the monitoring points very easy.

\subsection{Design of PMD-EC}

Traditionally, for the sake of supplying power safely, a room usually has a power distribution box within several no-fuse breakers. Hence, we designed PMD-EC to allow users to understand the power consumption status of a room by monitoring the current in the PDB. The functional blocks of PMD-EC are shown in Fig. 3. PMD-EC also has a plug, a breaker, PSU, VTU, and an

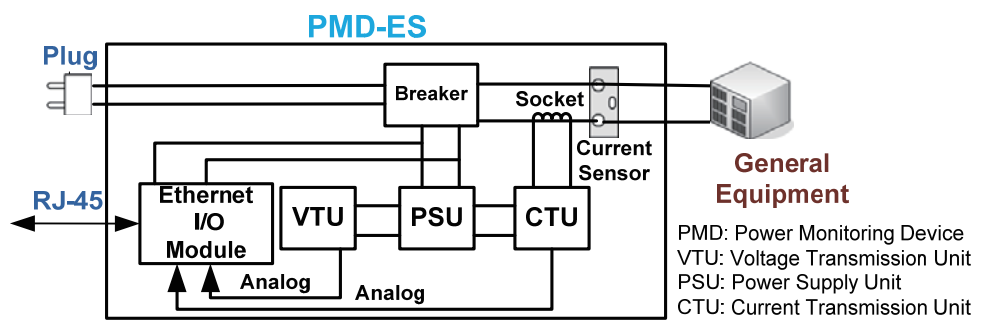

Fig. 2. (Color online) Functional blocks of PMD-ES.

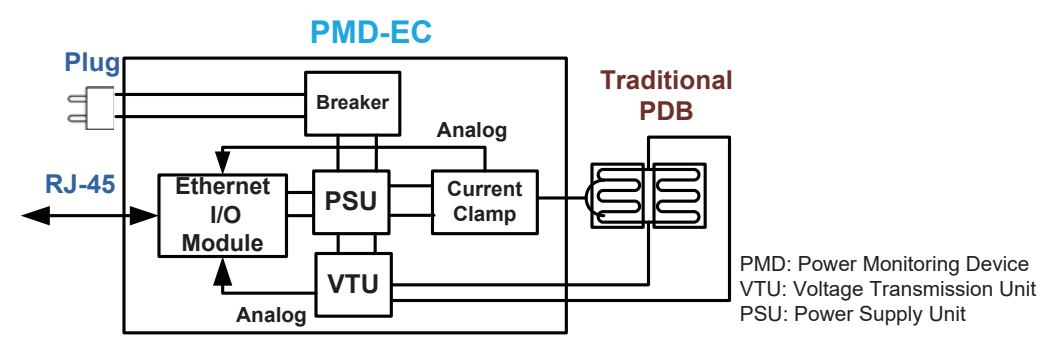

Fig. 3. (Color online) Functional blocks of PMD-EC. 
Ethernet I/O module. Unlike PMD-ES, PMD-EC has no socket but a current clamp that clasps on the power line inside the PDB to obtain the working current. In this way, users can compute the power consumption without reconstructing the PDB.

\subsection{Design of PMD-ER}

For convenience in power management, many PDBs are now equipped with digital meters. The digital meter usually has an RS-485 interface to connect with a PC through a Modbus protocol. ${ }^{(20)}$ In this study, we designed the PMD-ER to extend the communication distance and reduce the cost of the deployment of monitoring points. The functional blocks are shown in Fig. 4. PMD-ER has a plug, a breaker, a PSU, and an Ethernet I/O with an RS-485 module. Specifically, the Ethernet I/O with the RS-485 module can connect with the digital meter of a PDB to acquire power parameters using a Modbus protocol and then transmit them to the PMS via the Internet.

\subsection{Design of PMD-ZC}

The design of PMD-ZC is similar to PMD-EC and the functional blocks are shown in Fig. 5. The only difference between them is that PMD-ZC can transmit data over the air. To deploy the PMD-ZC in an environment without Internet infrastructure, PMD-ZC has a ZigBee reduced function device (RFD) that can transport data to a ZigBee coordinator wirelessly using the IEEE 802.15 .4 standard.

\subsection{Design of PMD-ZDC}

The design of PMD-ZDC allows the PMS to detect and compute abnormal conditions of the PDB and send a message to the PMS through the ZigBee protocol wirelessly. The functional

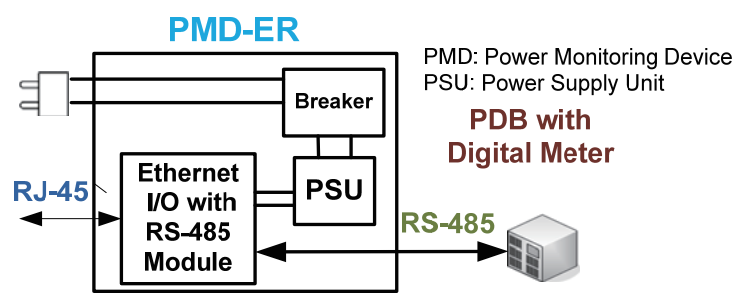

Fig. 4. (Color online) Functional blocks of PMD-ER.

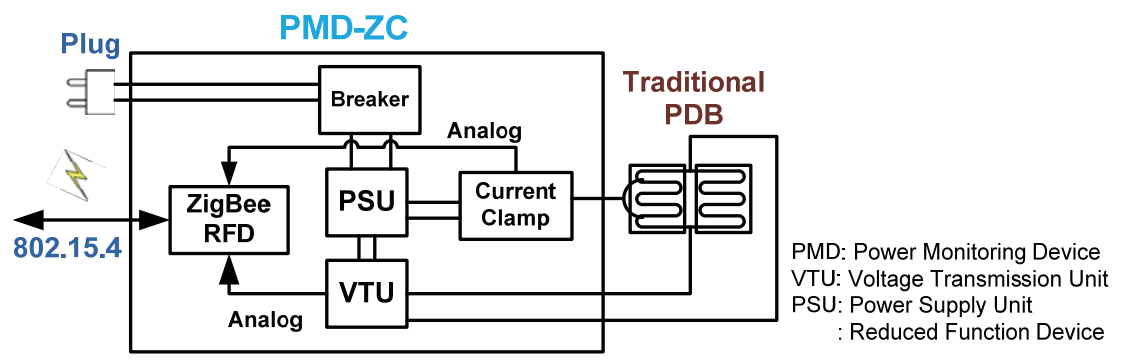

Fig. 5. (Color online) Functional blocks of PMD-ZC. 
blocks of the PMD-ZDC are shown in Fig. 6. Compared with PMD-ZC, PMD-ZDC has an extra DSP module connected with a ZigBee RFD through a universal asynchronous receiver/transmitter (UART) protocol. New power computing functions for specific requirements can be easily added in the PMD-ZDC by updating the DSP program code but leaving the hardware unchanged.

\subsection{Design of PMD-ZR}

The functional blocks of a PMD-ZR are shown in Fig. 7. PMD-ZR has a voltage adapter that can collect power parameters from digital meters of a PDB through a Modbus protocol and communicate with a ZigBee RFD through a UART protocol. The ZigBee RFD is responsible for transmitting data to the PMS wirelessly. Thus, PMD-ZR can be deployed in a PDB without Internet infrastructure.

In this research, we have designed six types of PMD that allow users to deploy them in different environments for various monitoring targets. We summarize their features in Table 1. PMD-ES, PMD-ES, and PMD-ER have a wired data transmission approach through an Ethernet protocol while PMD-ZC, PMD-ZDC, and PMD-ZR transmit data wirelessly via a ZigBee protocol. PMD$\mathrm{ES}$ is suitable for use in monitoring general equipment. In addition, PMD-EC, PMD-ZC, and PMD-ZDC can be deployed in a traditional PDB. Moreover, for the sake of monitoring a PDB

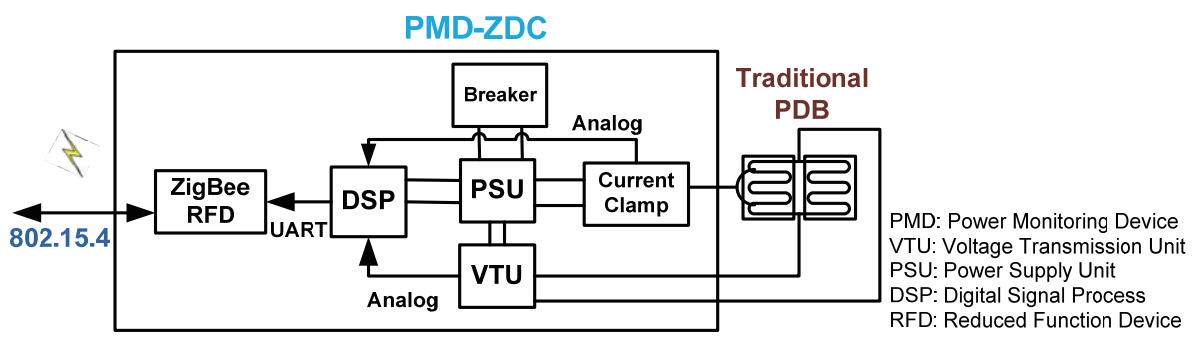

Fig. 6. (Color online) Functional blocks of PMD-ZDC.

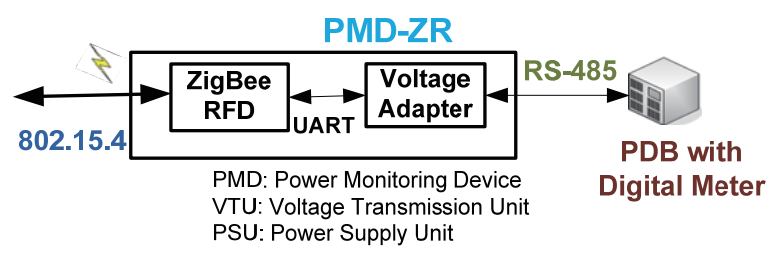

Fig. 7. (Color online) Functional blocks of PMD-ZR.

Table 1

Features of six types of PMD.

\begin{tabular}{lcccccc}
\hline & PMD-ES & PMD-EC & PMD-ER & PMD-ZC & PMD-ZDC & PMD-ZR \\
\hline \multirow{2}{*}{ Data transmission } & Wire & Wire & Wire & Wireless & Wireless & Wireless \\
& (Ethernet) & (Ethernet) & (Ethernet) & (ZigBee) & (ZigBee) & (ZigBee) \\
Target & General & Traditional & PDB with & Traditional & Traditional & PDB with \\
& equipment & PDB & digital meter & PDB & PDB & digital meter \\
Power signal process & $\times$ & $\times$ & $\times$ & $\times$ & $\circ$ & $\times$ \\
Load shedding control & $\times$ & $\times$ & $\times$ & $\times$ & $\circ$ & $\times$ \\
\hline
\end{tabular}


with digital meters, users can choose either PMD-ER or PMD-ZR. Specifically, PMD-ZDC possesses the capabilities of power signal processing and load shedding control that can be used in an unmanned storeroom to deal with abnormal situations of power consumption automatically.

\subsection{Design of real-time power-monitoring mechanism}

The design of a real-time power-monitoring mechanism allows the PMS to acquire the power parameters of the equipment through the PMD wirelessly using a ZigBee network. The procedure is shown in Fig. 8 and described as follows.

(1) The PMS finds the type of the PMD from the system database. If the type is PMD-ER or PMD$\mathrm{ZR}$, that means it is connected to a digital power meter, and the PMS converts the command into the Modbus format and sends it to the PMD via the Internet. If the PMD is PMD-ZR, PMD-ZC, or PMD-ZDC, it belongs to a ZigBee network, and the PMS sends the command to the ZigBee coordinator through an RS-232 interface. The ZigBee coordinator then passes the command to the PMD via the ZigBee network.

(2) After receiving the command from the PMS, the PMD acquires the power consumption information from the equipment.

(3) The PMD transmits the power parameters to the PMS through a wire or the wireless network that it owns.

(4) The PMS receives the data from the PMD. If the data is from a PMD-ER or PMD-ZR, the PMS parses the Modbus format to generate power parameters.

(5) The PMS displays the power parameters on the Web-based GUI.

\section{System Implementation and Integrated Test}

\subsection{System implementation}

By referring to the architecture in Fig. 1, we constructed a campus PMCS as an application paradigm to demonstrate the effectiveness of the proposed architecture. The implementation and

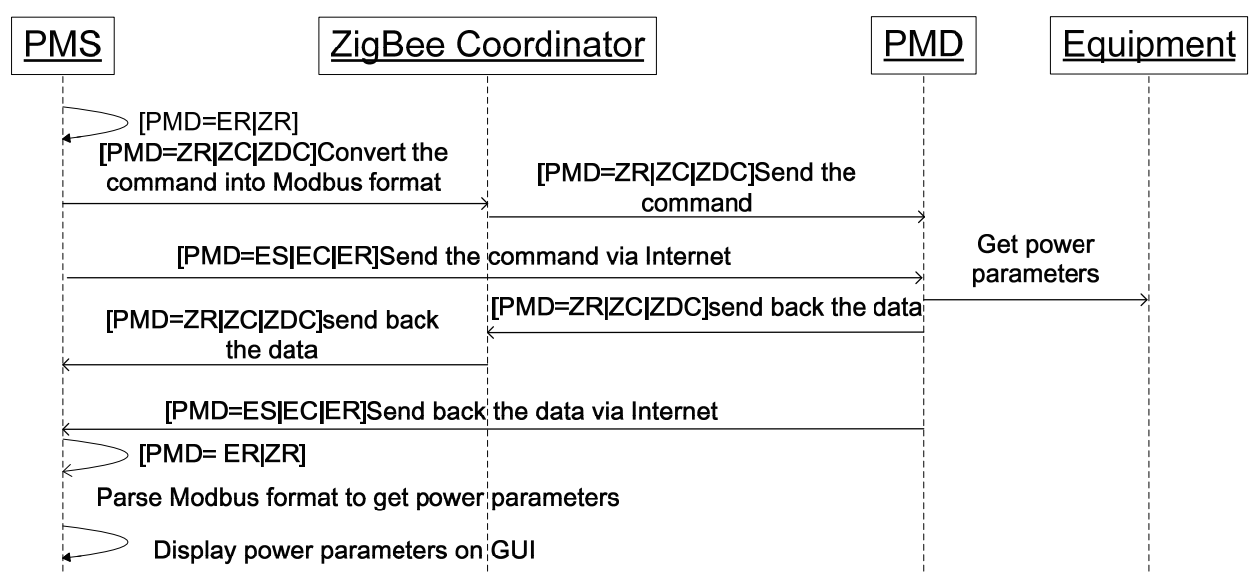

Fig. 8. Procedure for real-time power monitoring. 
integration test of the paradigm system is shown in Fig. 9. To implement the system, we used Windows 7 as the development platform. The system programs were written in $\mathrm{C \#}$ and ASP.NET, and Visual Studio was the programming tool. In addition, we exploited Microsoft Internet Information Services (IIS) as the Web server and employed Microsoft SQL Server 2008 to create the system database.

Regarding hardware requirements, a PC was used to construct the PMS, and a notebook was used as the client computer. We adopted the ADAM-6017 module of Advantech as the Ethernetready I/O module inside the PMD-ER, PMD-ES, and PMD-EC. ${ }^{(21)}$ In addition, as the current clamp inside the PMD-EC, PMD-ZC, and PMD-ZDC, we chose the DCT-100 of the MULTI Company. ${ }^{(22)}$ The ZigBee devices from Hua Heng Technology were adopted for the PMD-ZC, PMD-ZDC, and PMD-ZR. The DSP module from Texas Instruments Inc. ${ }^{(23,24)}$ was used to satisfy the power quality computing of the PMD-ZDC. We used a UART to the RS-485 Interface card from CuteDigi Technologies to meet the requirements of the PMD-ZR. ${ }^{(25)}$

Figure 9 shows the integration test of the paradigm power-monitoring system. The figure depicts the Web-based graphical user interfaces for authentication authorization, warning message transmission, user management, real-time power monitoring, and data management. The physical pictures of the six types of PMDs are also shown. The system provides the user interface based on the role of the user. Users can operate the system functions using browser-ready devices, such as computers, notebooks, PDAs, or smart phones after validation by the PMS. The real-time powermonitoring component has a Web-based chart that allows users to understand the maximum and minimum power consumption for a day or a week to help them adjust the working time of the equipment as well as to decrease the payment for power.

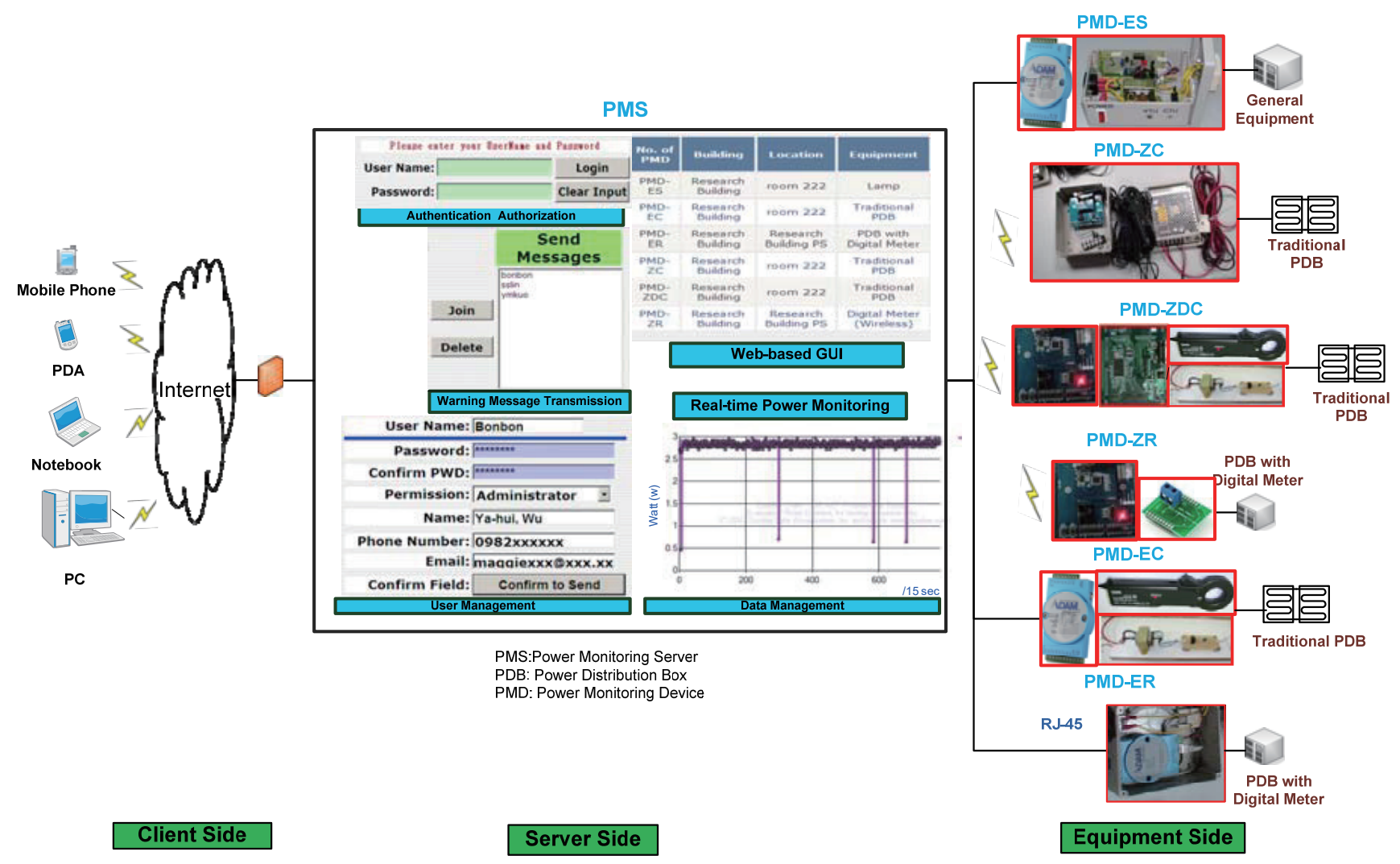

Fig. 9. (Color online) Implementation and integration test of the paradigm system. 


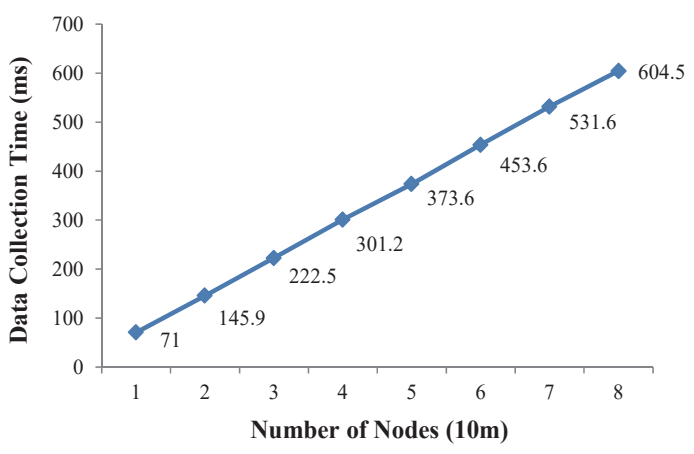

Fig. 10. (Color online) Data collection time at a distance of $10 \mathrm{~m}$.

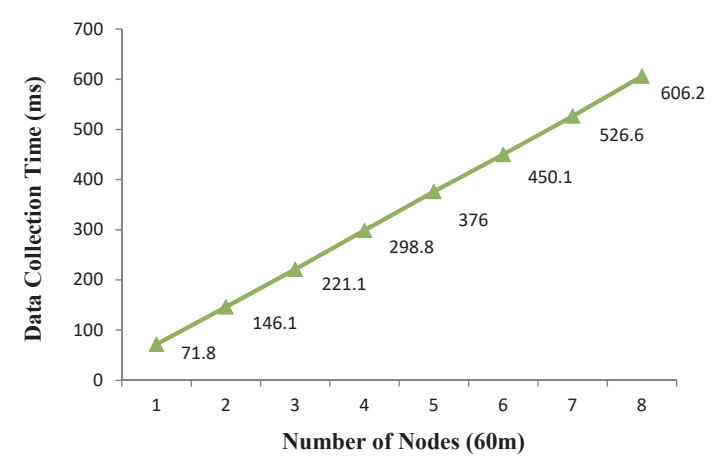

Fig. 12. (Color online) Data collection time at a distance of $60 \mathrm{~m}$.

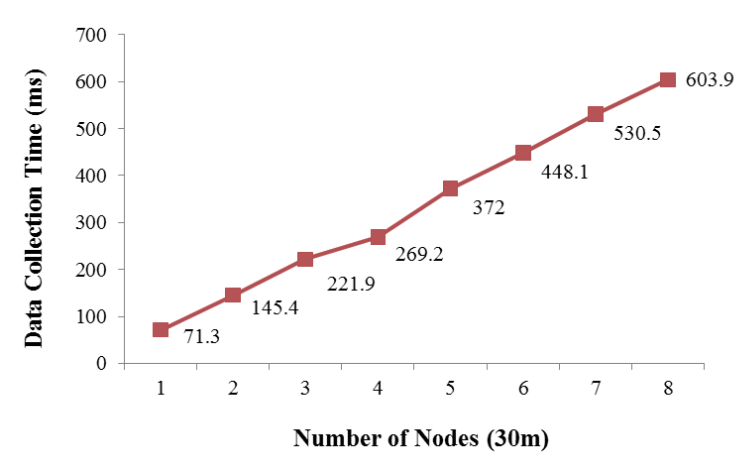

Fig. 11. (Color online) Data collection time at a distance of $30 \mathrm{~m}$.

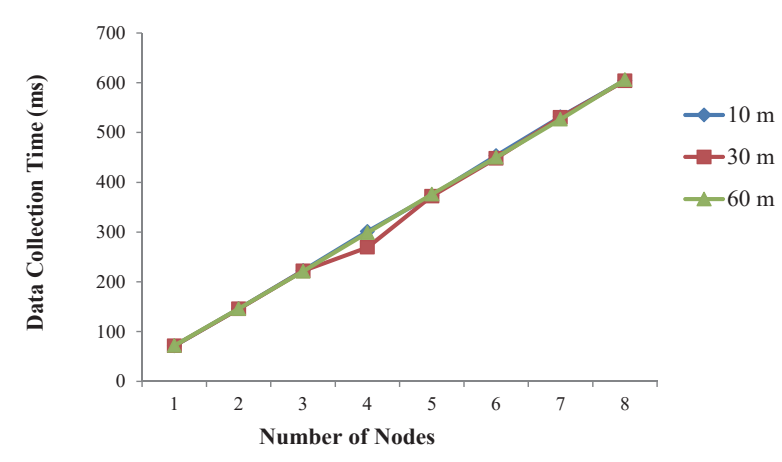

Fig. 13. (Color online) Data collection time at different distances.

\subsection{Evaluation of wireless transmission}

To evaluate the wireless transmission efficiency of the PMS system, we deployed the data collection schemes mentioned in Sect. 3.7 into the ZWSN as a test. The transmission distances between the ZCR and ZigBee end devices were 10,30, and $60 \mathrm{~m}$. The data collection task was executed from 1 to 8 ZigBee end devices. The test results are shown in Figs. 10-12. A positive correlation was found between the data collection time and the number of ZigBee end devices. We summarize the test result in Fig. 13. We observe that there were no significant relationships between the different distances. The PMS can collect the data from 8 ZigBee end devices deployed at a distance of $60 \mathrm{~m}$ in about $0.6 \mathrm{~s}$. The test results show that the response time can fulfill the requirements of real-time power-monitoring systems.

\section{Conclusions}

To diminish the drawbacks existing in current PMCSs, we developed a novel PMCS architecture based on Internet and wireless sensor networks, which possesses the advantages of easy deployment and applicability to various monitoring points. First, we summarized the 
requirements by reviewing the literature about current PMCSs. Accordingly, in this paper, we propose a novel PMS architecture, including consideration of the equipment side, the server side, and the client side. For the equipment side, we designed six kinds of PMDs, three using Ethernet interfaces and the others using ZigBee wireless sensor networks, which allow users to deploy them for various monitoring targets in different environments. On the server side, several functional components, such as real-time monitoring, data management, alarm message transmission, and Web-based GUI were developed into the PMS to provide the necessary capabilities for power management including monitoring, recording, displaying, and managing the power parameters of the monitoring targets. In addition, the PMS can detect abnormal situations of the targets and send alarm messages to responsible users. Users can operate the PMS functions using any devices within a browser, such as desktops, laptops, or tablets, via the Internet from any location.

Finally, a paradigm power-monitoring system was constructed to monitor the power consumption of the campus at Chung Cheng Institute of Technology, National Defense University, to validate the feasibility and practicality of the proposed PMS architecture. The results of this research will be a useful reference for developing PMSs in the future.

\section{References}

1 L. Huang and L. Wang: Proc. CAC (IEEE, 2015) p. 1642.

2 Y. Fan and H. Chen: Proc. CDCSS (IEEE, 2013) p. 295.

3 R. Makwana, J. Baviskar, N. Panchal, and D. Karia: Proc. ICEETS (IEEE, 2013) p. 34.

4 G. L. Zheng, Y. C. Gao, and L. J. Wang: Proc. APPEEC (IEEE, 2010) p. 1.

5 Lin, H: Proc. ICSMC (IEEE, 2005) p. 2867.

6 M. Zhang and K. C. Li: Proc. ICISE (IEEE, 2009) p. 1577.

7 C. Chengyi, Z. Guanna, and J. Minglu: Proc. ICSPS (IEEE, 2010) p. 373.

8 F. Benzi, N. Anglani, E. Bssi, and L. Frosini: IEEE Trans. IE 58 (2011) 4487.

9 C. Tatsiopoulos and A. Ktena: Proc. SSIP (IEEE, 2009) p. 1.

10 O. Mirabella and M. Brischetto: IEEE Trans. IM 60 (2011) 398.

11 T. S. Choi, K. R. Ko, S. C. Park, Y. S. Jang, and Y. T. Yoon: Proc. TDCE (IEEE, 2009) p. 1.

12 K. Gill, S. H. Yang, F. Yao, and X. Lu: IEEE Trans. CE 55 (2009) 422.

13 A. Iwayemi, P. Yi, P. Liu, and C. Zhou: Proc. ISGT (IEEE, 2010) p. 1.

14 D. M. Han and J. H. Lim: IEEE Trans. CE 56 (2010) 1403.

15 J. Han, H. Lee, and K. R. Park: IEEE Trans. CE 55 (2009) 1.

16 S. W. Luan, J. H. Teng, S. Y. Chan, and L. C. Hwang: Proc. PEDS (IEEE, 2009) p. 661.

17 X. Chen and X. Jiang: Proc. ECE (IEEE, 2010) p. 2671.

18 I. Hong and S. Park: Proc. ICCE (IEEE, 2011) p. 311.

19 T. Itoh, Y. Zhang, M. Matsumoto, and R. Maeda: Proc. ICS (IEEE, 2010) p. 572.

20 D. Suhartono, A. Wibowo, S. Wiguna, and R. Saleh: Procedia Eng. 50 (2012) 426.

21 Advantech Co. Ltd.: http://www.advantech.tw (accessed June 2016).

22 Multi Company: http://www.multimic.com (accessed June 2016).

23 Hua Heng Technology Ltd.: http://www.hhnet.com.tw (accessed June 2016).

24 Texas Instruments Inc.: http://www.ti.com (accessed June 2016).

25 CuteDigi Technologies, Inc.: http:// www.cutedigi.com (accessed June 2016). 